\title{
Silencing of the long non-coding RNA NEAT1 suppresses glioma stem-like properties through modulation of the miR-107/CDK6 pathway
}

\author{
XIAOLI YANG ${ }^{1}$, ZHENG XIAO $^{2}$, XIAOWEI DU $^{1}$, LINA HUANG $^{1}$ and GANQIN DU ${ }^{1}$ \\ Departments of ${ }^{1}$ Neurology and ${ }^{2}$ Neurosurgery, The First Affiliated Hospital, and College of Clinical Medicine \\ of Henan University of Science and Technology, Luoyang, Henan 471003, P.R. China
}

Received May 19, 2016; Accepted June 29, 2016

DOI: $10.3892 /$ or.2016.5266

\begin{abstract}
Developing novel strategies against glioma remains a significant challenge. Long non-coding RNA nuclear paraspeckle assembly transcript 1 (NEAT1) significantly contributes to the progression of many human cancers, while glioma stem cells (GSCs) are believed to be responsible for tumor progression. However, whether NEAT1 alters the stem-like properties of GSC cells remains unknown. Using microbeads, $\mathrm{CD}_{133^{+}}$cells were isolated and were found to possess glioma stem cell properties. Using siRNA, NEAT1 was knocked down in the sorted CD133+ U87 glioma cells. We found higher NEAT1 RNA expression in CD133+ human glioma primary culture stem cells and CD133 ${ }^{+}$U87 cells via RT-PCR. Moreover, NEAT1 knockdown in the CD133+ U87 cells resulted in decreased colony formation, increased G1 cell cycle arrest and apoptosis. In addition, these effects were accompanied by miR-107 activation and inactivation of CDK6 protein. These results strongly suggest that NEAT1 knockdown suppresses stem-like properties in glioma cells by modulating the miR-107CDK6 pathway. Targeting NEAT1 may provide a novel therapeutic opportunity for developing a relapse-free treatment of glioma patients.
\end{abstract}

\section{Introduction}

Glioma is the most common primary malignant tumor in the central nervous system, and is associated with poor prognosis and rapid mortality (1). It accounts for 30-40\% of all intracranial tumors (2). Although the standard treatment for glioma is based on the combination of surgery, chemotherapy and radiation therapy, the relapse rate is still very high (3).

Correspondence to: Dr Ganqin Du, Department of Neurology, The First Affiliated Hospital, and College of Clinical Medicine of Henan University of Science and Technology, Luoyang, Henan 471003, P.R. China

E-mail: ganqindu@163.com

Key words: glioma, NEAT1, glioma stem cells, miR-107, CDK6
Based on certain pathological characteristics, gliomas are classified into 4 grades (WHO grade I, II, III and IV). Grade I or II tumors are low grade astrocytomas, grade III tumors are anaplastic astrocytomas, and grade IV tumors are glioblastoma multiforme (4). To date, the median survival time of patients with grade IV glioblastoma multiforme is only 14.7 months (5). A dilemma in this area is further compounded by the fact that patients with glioblastoma (WHO grade IV) account for 50\% of the new cases of malignant CNS tumors (5). Recurrence and invasion of the primary tumor are thought to be key contributors to the incurable nature of glioma (2). Glioma stem cells (GSCs) are a subgroup of glioma cells having the ability to self-renew and to differentiate into mature tumor cells $(6,7)$. Accumulating evidence suggests that GSCs play an important role in tumorigenesis, treatment resistance and tumor recurrence $(1,7)$. Thus, therapy targeting GSCs is of critical importance in the development of relapse-free treatment for glioma patients.

Recently, long non-coding RNAs (lncRNAs), which represent non-protein coding transcripts longer than 200 nucleotides, have been implicated in several cellular functions, including cell proliferation, apoptosis, transformation, metastasis and differentiation $(8,9)$. Accumulated evidence has proposed that RNA interference holds great promise for tumor therapy. Among these lncRNAs, nuclear enriched abundant transcript 1 (NEAT1) has been advocated as an important mediator of many tumor cells. NEAT1 expression levels have been found to be related with the pathologic grade in many types of cancer, including esophageal squamous cell carcinoma (10), colorectal (11) and prostate cancer, and gliomas $(12,13)$. Furthermore, NEAT1 knockdown was also found to induce apoptosis in B-cell lymphoma and LSCC cells $(14,15)$. In the glioma research field, knockdown of NEAT1 was reported to reduce glioma cell proliferation in vitro (16). Since studies focusing on the NEAT1 function in glioma are limited, whether NEAT1 is capable of targeting GSCs remains unclear. A better understanding of the precise function of NEAT1 on GSCs is important to improve current therapies and to design new treatment modalities.

In our previous study, we found that NEAT1 knockdown plays a crucial regulatory role in proliferation, apoptosis and metastasis of esophageal squamous cell carcinoma (10). We 
hypothesized that NEAT1 plays a regulatory role in GSCs. In the present study, we provide experimental evidence to support this hypothesis and demonstrate a functional role of NEAT1 in GSCs. We demonstrated that NEAT1 knockdown induced G1 cell cycle arrest, and apoptosis while reducing proliferation in GSCs. The present study proposes that the targeting of NEAT1 may represent a novel and important therapeutic strategy for the treatment of glioma.

\section{Materials and methods}

Patients and surgical specimens. Tissue samples from patients with gliomas were used in the present study. Diagnostic criteria for gliomas were preoperative magnetic resonance imaging (MRI) and postoperative histopathological findings. All patients received surgery between May 2015 and December 2015 at the Department of Neurosurgery, The First Affiliated Hospital of Henan University of Science and Technology (Luoyang, China). There were 29 patients with gliomas (18 men, 11 women; age range $45-69$ years, mean age $51.50 \pm 16.00$ years). For each sample, the tissue was dissected. One half was sent to the laboratory for primary culture, protein or RNA extraction, and the other half was fixed and embedded in paraffin for histology and immunohistochemisty studies. The study protocol was approved by the local Ethics Committee of the Henan University of Science and Technology and informed consent was obtained from all patients in the present study.

Cell lines and culture protocols. The U87 human glioblastoma cell line was purchased from the American Type Culture Collection (ATCC; Manassas, VA, USA). Glioma primary cultured cells were prepared in our laboratory as follows. Resected brain glioma tissues were immediately send to the laboratory by placing them in $0.9 \% \mathrm{NaCl}$. Samples were digested by incubation in $0.1 \%$ type I collagenase (SigmaAldrich, St. Louis, MO, USA) in phosphate-buffered saline (PBS) at $37^{\circ} \mathrm{C}$ for $2 \mathrm{~h}$, and then filtered using a $40-\mu \mathrm{m}$ steel mesh. The cell suspensions were cultured in the following medium. For regular cell maintenance, the cells were cultured in Dulbecco's modified Eagle's medium (DMEM) containing $10 \%$ fetal bovine serum (FBS; Gibco, Life Technologies, Carlsbad, CA, USA) and $100 \mathrm{U} / 100 \mu \mathrm{g} / \mathrm{ml}$ penicillin-streptomycin in a humidified incubator at $37^{\circ} \mathrm{C}$ with $5 \% \mathrm{CO}_{2}$.

To induce stem cell proliferation and negatively select against differentiated cells, the stem cells isolated from U87 and primary cultured cells were cultured in DMEM-F12 supplemented with $20 \mathrm{ng} / \mu 1 \mathrm{hEGF}, 20 \mathrm{ng} / \mu \mathrm{lbFGF}$ (both from Gibco), 1X B27 and $100 \mathrm{U} / 100 \mu \mathrm{g} / \mathrm{ml}$ penicillin-streptomycin in a humidified incubator at $37^{\circ} \mathrm{C}$ with $5 \% \mathrm{CO}_{2}$.

Isolation and identification of glioma stem cells. The cell suspensions were collected and washed with PBS and incubated with magnetic microbeads conjugated with the anti-CD133 antibody (Miltenyi Biotec, Cambridge, MA, USA). The bead-bound $\left(\mathrm{CD} 133^{+}\right)$and unbound cells (CD133-) were separated using the QuadroMACS ${ }^{\mathrm{TM}}$ Separation Unit (Miltenyi Biotec). The purity of the isolated CD133 ${ }^{+}$cells was confirmed by flow cytometric analyses. The isolated CD133+ cells were then cultured in stem cell media.
Neurospheres. Briefly, $1 \times 10^{3} \mathrm{CD}_{133^{+}}$cells were seeded into 6-well ultra-low attachment culture plates (Corning Inc., Corning, NY, USA) with DMEM-F12 supplemented with $20 \mathrm{ng} / \mu \mathrm{l} \mathrm{hEGF}, 20 \mathrm{ng} / \mu \mathrm{l} \mathrm{bFGF}$ (both from Gibco), $1 \mathrm{X}$ B27 and $100 \mathrm{U} / 100 \mu \mathrm{g} / \mathrm{ml}$ penicillin-streptomycin. Culture medium was replaced every 4 days. Glioma spheres derived from one mother cell in the subsphere forming assay were mechanically digested into single cells that were seeded into standard medium for differentiation (DMEM with $10 \%$ of FBS). Then, the cells were analyzed at different time points.

Virus transduction. The siNEAT1 and the control lentiviruses (GFP-lentivirus) were constructed by GeneCopoeia-FulenGen (Shanghai, China). The sequences of siNEAT1 were as follows: 5'-GUGAGAAGUUGCUUAGAAACUUUCC-3'. CD133+ GSC cells were transduced as follows. The lentiviruses were diluted in complete medium incubated with the cells for $12 \mathrm{~h}$ at $37^{\circ} \mathrm{C}$. Next, the cells were incubated with freshly prepared Polybrene-DMEM for $24 \mathrm{~h}$. Cells were selected in medium containing $5 \mu \mathrm{g} / \mathrm{ml}$ puromycin (Sigma-Aldrich) for $48 \mathrm{~h}$.

Soft agar colony formation assays. The proliferation of the $\mathrm{CD}_{133^{+}}$GFP control and $\mathrm{CD}_{133^{+}}$siNEAT1 cells was measured using the soft agar colony formation assays. Briefly, $\sim 1 \times 10^{5}$ cells were mixed with medium containing $0.4 \%$ agar and were spread on top of a bottom agar layer $(0.8 \%$ agar in growth medium). Cells were grown for 2 weeks, and colonies were counted and photographed. The diameter of the colonies was measured using ImageJ software.

Cell cycle analysis by flow cytometry. Cells were collected and fixed with cold ethanol at $4^{\circ} \mathrm{C}$ for $1 \mathrm{~h}$ before being stored at $-20^{\circ} \mathrm{C}$. Fixed cells were washed and resuspended in $1 \mathrm{ml}$ PBS containing $50 \mu \mathrm{g} / \mathrm{ml} \mathrm{RNase} \mathrm{A}$ and $50 \mu \mathrm{g} / \mathrm{ml}$ ethidium bromide. After incubating for $20 \mathrm{~min}$ at $37^{\circ} \mathrm{C}$, the cells were analyzed for DNA content by flow cytometry (FACSCalibur; Becton-Dickinson Immunocytometry Systems, San Jose, CA, USA). For each sample, 20,000 events were acquired, and cell cycle distribution was determined using cell cycle analysis software (ModFit LT for Mac version 3.0).

Apoptosis assay by flow cytometry. The cells were washed twice with cold $10 \mathrm{mM}$ PBS and resuspended in $1 \mathrm{X}$ binding buffer (BD Biosciences, San Jose, CA, USA) at a density of $1 \times 10^{6}$ cells $/ \mathrm{ml}$. Cells were stained with Annexin V/APC and propidium iodide (PI), using the Annexin V apoptosis detection kit (BD Biosciences).

Quantitative real-time PCR. Total RNA containing miRNA was extracted from cells using a Qiagen miRNeasy Mini kit (Qiagen, Mississauga, ON, USA) following the manufacturer's instructions. cDNA was reverse transcribed using All-in-One miRNA RT-qPCR kit (GeneCopoeia, Rockville, MD, USA). Real-time PCR was performed with gene-specific primers using a Bio-Rad CFX96 Real-Time PCR System (Bio-Rad, Hercules, CA, USA). Primer sequences used in the present study were the following: NEAT1 forward, 5'-CTTCCTCCCT TTAACTTATCCATTCAC-3' and reverse, 5'-CTCTTCCTCC ACCATTACCAACAATAC-3'; miR-107 forward, 5'-ATGATG AGCAGCATTGTACAGG-3' and reverse, 5'-GCAGGGTCC 

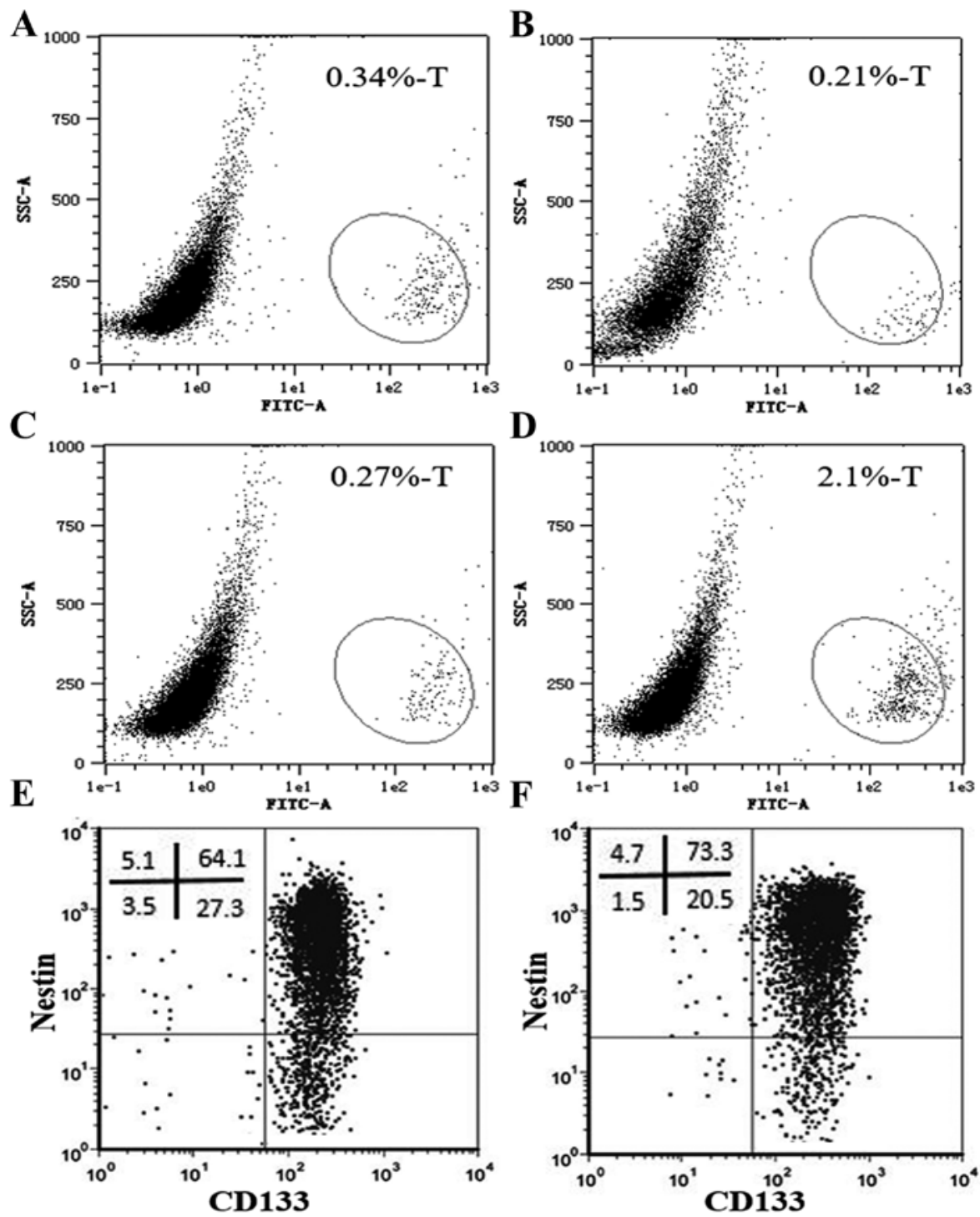

Figure 1. The percentage of CD133 or nestin-positive cells in the primary human glioma tissue culture and U87 human glioma cells. The representative flow cytometric dot-plots of CD133+ cells in the primary culture cells of human glioma WHO grade IV tissues from patient (A) 1 , (B) 2 , (C) 3 and (D) in the U87 human glioma cell line. The representative cytometric dot-plots of nestin and CD133 expression on CD133 ${ }^{+}$stem like cells isolated from primary culture cells of (E) human glioma tissues and (F) the U87 human glioma cell line.

GAGGTATTC-3'. GAPDH was used as an NEAT1 internal control and U6 small nuclear RNA was used as an miR-107 internal control. Samples were run in triplicate.

Western blot analysis. Western blotting was performed as previously described $(17,18)$. Protein extracts were electrophoresed on a $12 \%$ SDS-PAGE and transferred to nitrocellulose membrane. The following antibodies were used: rabbit antiCDK6 (1:1,000; Abcam, Cambridge, MA, USA) and mouse anti-actin (1:3,000; Cell Signaling Technology, Danvers, MA, USA). Signals were visualized using enhanced chemiluminescence (ECL) reagents (GE Healthcare, Barrington, IL, USA) and captured with a FluorChem imager from Bio-Rad.

Statistical analysis. Statistical analysis was conducted using the SPSS 16.0 software. The results are presented as mean \pm SD and analyzed with the Student's t-test. $\mathrm{P}<0.05$ was denoted as statistically significant.

\section{Results}

Isolation and identification of GSCs. In the present study, we first isolated GSCs from human glioma tissues. As CD133 is the most accredited marker for GSCs (19-22), CD133 was employed to identify GSCs in the present study. The resected brain glioma tissues were primary cultured by collagenase I digestion method. We first analyzed the ratio of $\mathrm{CD} 133^{+}$cells in the primary cultured human glioma (Fig. 1A-C) and U87 cells (Fig. 1D). As shown in Fig. 1A-C, the ratio of CD133+ cells in the primary cultured human WHO grade IV glioma cells was very low and varied greatly in the 3 different patient tissues. In order to increase cell homogeneity, the U87 human glioma cells and the primary cultured human glioma cells were used to isolate GSCs (Fig. 2A and B). We primary cultured human glioma and U87 cells in serum-free media containing epidermal growth factor and basic fibroblast growth factor to preferentially induce stem cell proliferation and negatively 
A

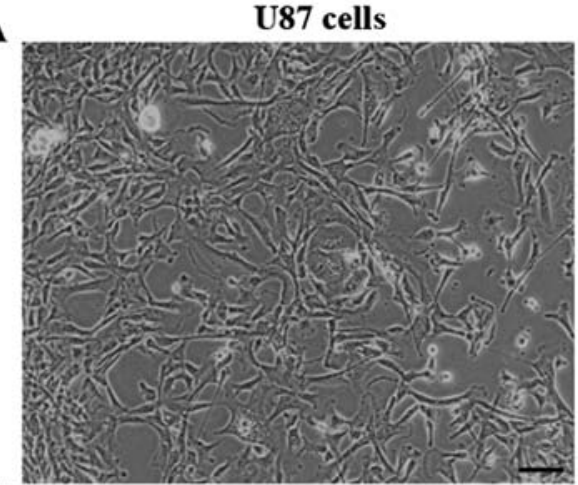

C

$\mathbf{E}$
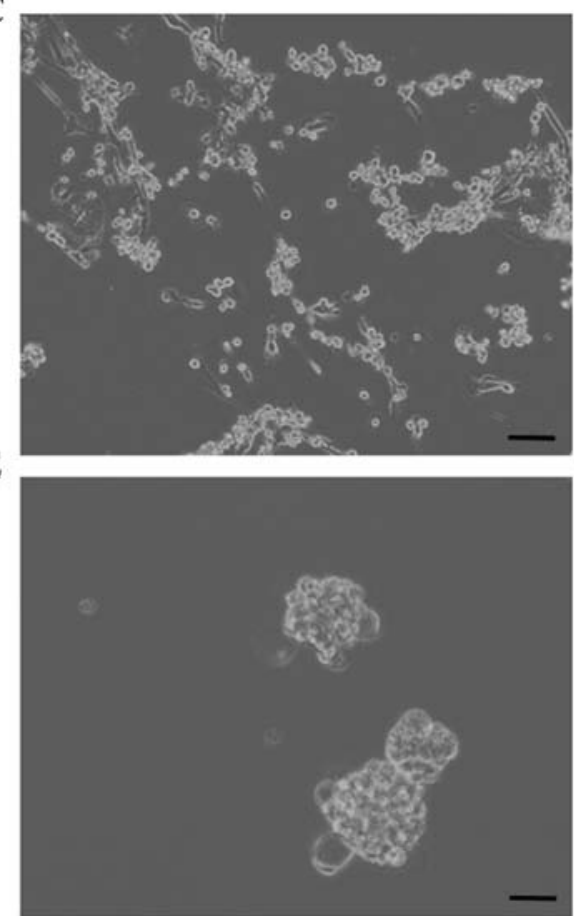

B

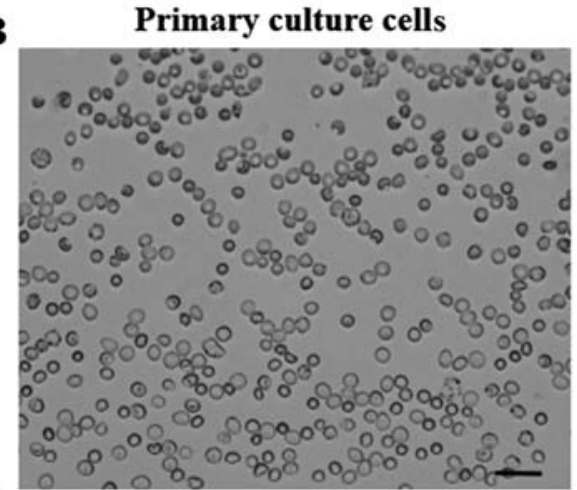

D

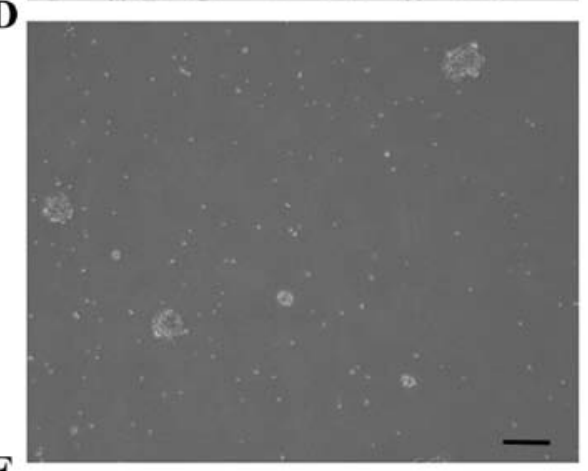

F

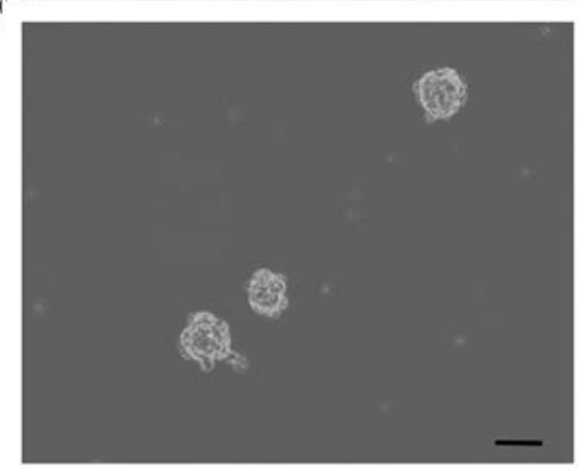

Figure 2. Representative microscopic analysis of cultured cells. (A) CD133+ U87 cell line and (B) CD133 ${ }^{+}$primary cultured human glioma cells. Formation of neurospheres in (C) U87 cells and (D) primary cultured glioma cells after 3 days in serum-free media containing EGF and bFGF medium. Images of neurospheres generated in CD133 ${ }^{+}$cells in (E) U87 cells and (F) primary cultured glioma cells on day 7. Scale bar, $100 \mu \mathrm{m}$.

select against differentiated cells (Fig. 2C and D). As shown in our previous studies $(17,23)$, the immunomagnetic bead sorting technique was used to isolate cancer stem cells from human glioma primary cultured cells and U87 glioma cell line in the present study.

After isolation, the purity of the isolated $\mathrm{CD}_{133^{+}}$cells was confirmed (Fig. 1E and F) and the glioma stem cell properties were identified. Most of the isolated cells also displayed nestin expression (Fig. 1E and F). The neurospheres emerged after one week of culture (Fig. 2E and F). Furthermore, the neurospheres had high frequencies of secondary neurosphere formation. Moreover, when spheroid cells were cultured under differentiation conditions for 3 days, the neurosphere cells were adherent to the culture plate and grew in monolayer. On the basis of this evidence and morphological aspect, most of the $\mathrm{CD}_{133^{+}}$isolated cells displayed glioma stem cell properties: self-renewal and self-differentiation abilities.

$\mathrm{CD} 133^{+}$human glioma primary culture stem cells display higher NEAT1 expression levels. NEAT1 has been reported to be correlated with higher WHO grade human glioma tissues (12). Therefore, in the present study, we examined the NEAT1 expression levels in 29 human glioma tissues (5 WHO II, 11 WHO III, 13 WHO IV grade) using PCR. The WHO III and IV glioma tissues displayed higher NEAT1 levels than the WHO II grade tissues (Fig. 3A). However, contrary to what we expected, there was no significant difference between the WHO III and IV glioma tissues, which was inconsistent with previous studies $(12,16,24)$. Next, we determined the NEAT1 expression levels in CD133 ${ }^{+}$human GSCs and human glioma primary culture cells. We found that the CD133+ human GSCs displayed significantly higher NEAT1 levels than the human glioma primary culture cells. Being similar to the human tissues, the $\mathrm{CD} 133^{+} \mathrm{U} 87$ cells displayed higher NEAT1 levels than the regular U87 cells (Fig. 3B).

NEAT1 knockdown reduces proliferation while induces cell cycle arrest and apoptosis of $\mathrm{CD} 133^{+}$U87 cells. In order to define the role of NEAT1 in the CD133+ GSCs, we transfected the $\mathrm{CD} 133^{+}$human GSCs with siNEAT1 and scramble siRNA. 
A

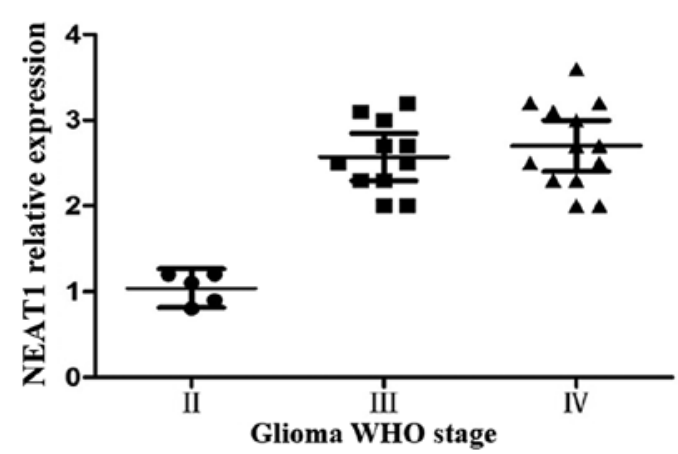

B

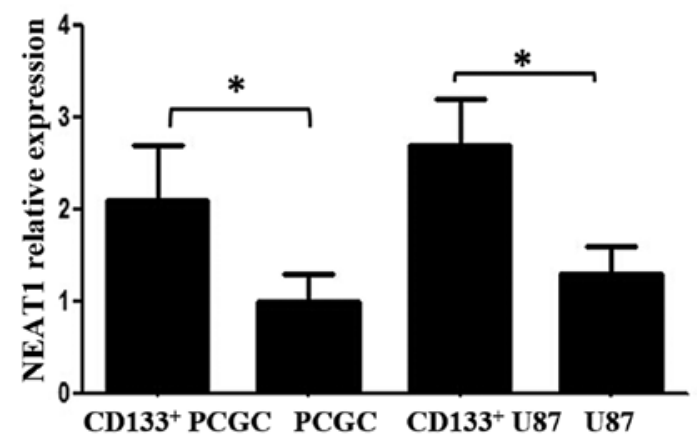

Figure 3. NEAT1 expression levels in glioma. (A) Expression of NEAT1 in glioma tissues from WHO stage II-IV. (B) Expression of NEAT1 in CD133+ human primary cultured glioma cells (CD133 ${ }^{+}$PCGC), PCGC, CD133 ${ }^{+} \mathrm{U} 87$ and regular U87 cells; ${ }^{*} \mathrm{P}<0.05$.

A

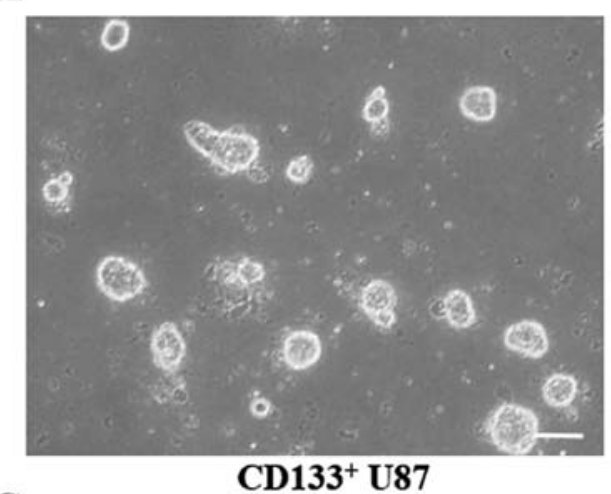

C

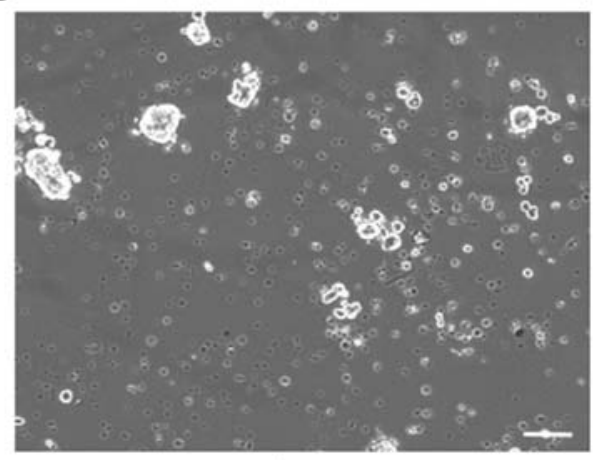

$\mathrm{CD133}^{+}$U87/siNEAT1
B

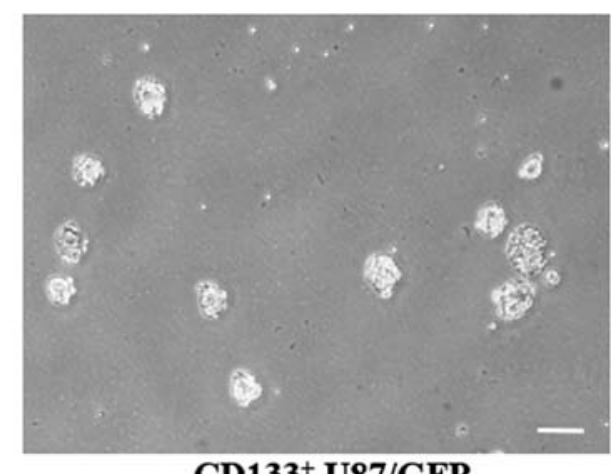

D

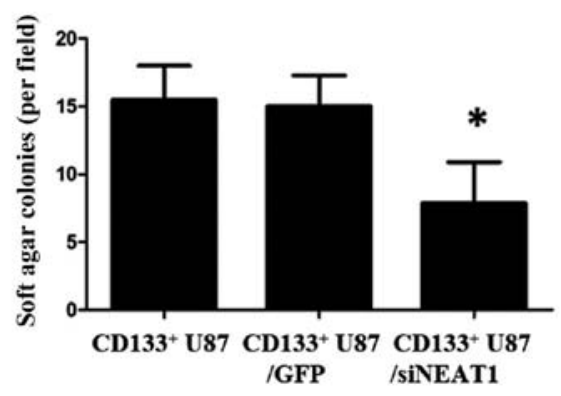

Figure 4. NEAT1 knockdown reduces the proliferation of CD133 ${ }^{+} \mathrm{U} 87$ cells. Representative images of soft-agar colony formation assays of (A) CD133 ${ }^{+} \mathrm{U} 87$, (B) $\mathrm{CD} 133^{+}$U87/GFP and (C) CD133+ U87/siNEAT1 cells. (D) The bar graph shows that NEAT1-knockdown CD133 ${ }^{+}$U87 cells formed less colonies compared to the control CD133' 887 and CD133+ U87/GFP cells. Scale bar, $100 \mu \mathrm{m} ;{ }^{*} \mathrm{P}<0.05$.

However, we found that the CD133+ human GSCs from different tissues displayed variable transfection efficiency. Due to this reason, the $\mathrm{CD} 133^{+} \mathrm{U} 87$ cells were transfected and the efficiency was confirmed. Then, we conducted the soft agar colony formation assays. We found that the proliferation of NEAT1-knockdown CD133 ${ }^{+}$U87 cells was significantly lower than the relative control group (Fig. 4). To further investigate the influence of NEAT1 on the GSCs, flow cytometry was also used to examine the cell cycle distribution and cell apoptosis. We found an increased apoptosis rate and an increased G1 phase accumulation in the NEAT1-knockdown CD133+
U87 cells compared with normal CD133 ${ }^{+} \mathrm{U} 87$ and control $\mathrm{CD}_{133^{+}} \mathrm{U}$ 87/GFP cells (Fig. 5A and B).

NEAT1 regulates CDK6 expression by modulating miR-107. Recent study have shown that NEAT1 may regulate miR-107, which in turn directly modulates CDK6 expression by binding to the miR-107 seed complementary site located in 3'UTR of CDK6 (15). CDK6 is known to be an important regulator of cell cycle progression, modulating cell cycle G1 phase progression and $\mathrm{G} 1 / \mathrm{S}$ transition (25). In order to further explore the mechanistic base of NEAT1 in GSCs, we verified the miR-107 RNA 


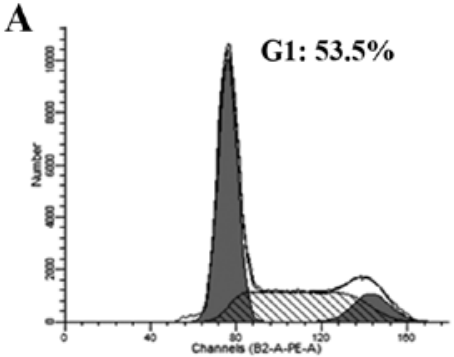

B

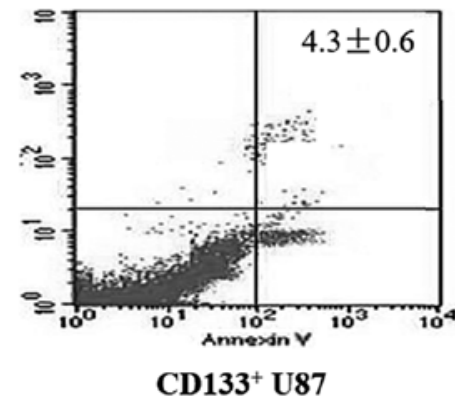

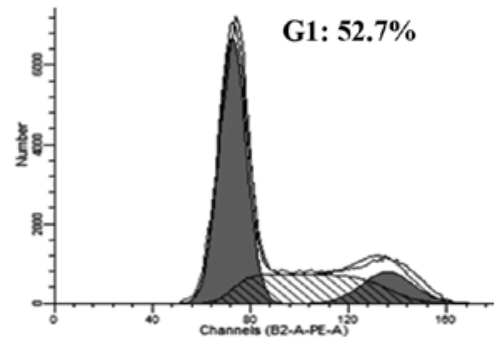

$\mathrm{CD} 133^{+}$U87/GFP

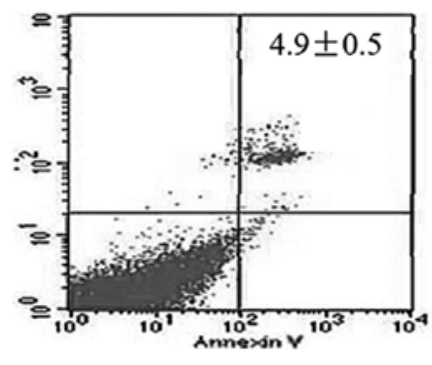

$\mathrm{CD}^{133}{ }^{+}$U87/GFP

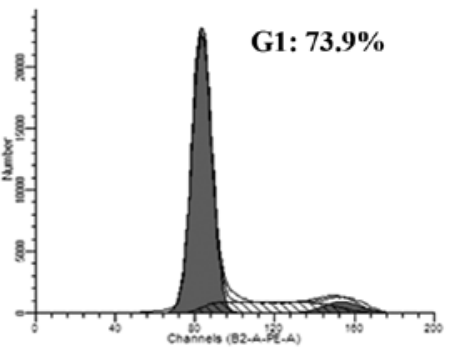

$\mathrm{CD}^{133}{ }^{+}$U87/siNEAT1

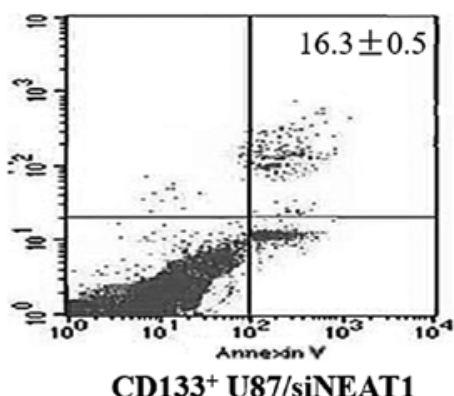

Figure 5. NEAT1 knockdown induces cell cycle arrest and apoptosis in CD133 ${ }^{+}$U87 cells. (A) Flow cytometric cell cycle analysis showed increased G1 phase accumulation in the NEAT1-knockdown CD133+ U87 cells. (B) Flow cytometric apoptosis analysis showed an increased apoptosis rate in the NEAT1-knockdown CD133 ${ }^{+}$U87 cells compared with the normal CD133+ ${ }^{+} 87$ and CD133 ${ }^{+}$U87/GFP cells.

A

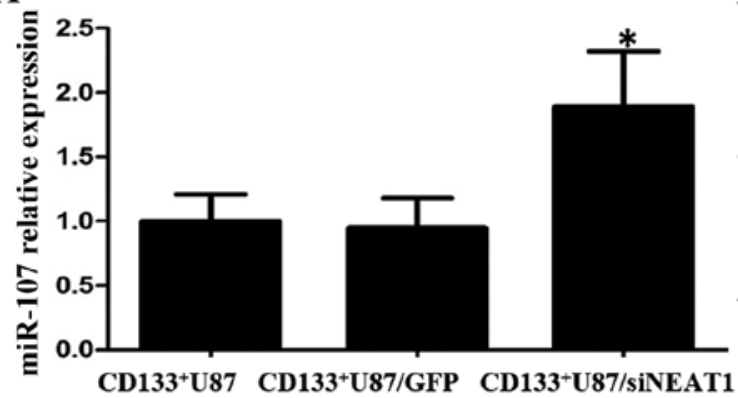

B

CDK6

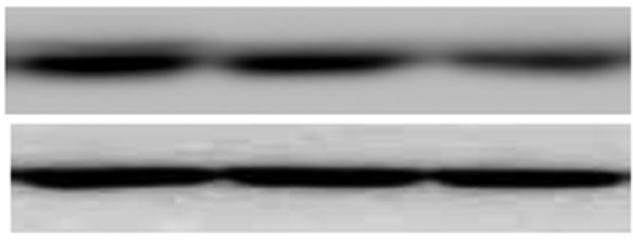

CD133+U87 CD133+U87/GFP CD133+U87/siNEAT1

Figure 6. NEAT1 regulates CDK6 expression through modulation of miR-107. (A) miR-107 expression was analyzed in the CD133 ${ }^{+}$U87 cells infected with siNEAT1, control vector and negative control by real-time-PCR. (B) CDK6 protein expression was analyzed in the CD133+ U87 cells infected with the siNEAT1, control vector and negative control by western blotting; ${ }^{*} \mathrm{P}<0.05$.

and CDK6 protein levels in NEAT1-knockdown CD133+ U87 cells. We found increased miR-107 RNA levels and decreased CDK6 protein levels in the NEAT1-knockdown CD133+ U87 cells compared with these levels in the normal $\mathrm{CD}_{133^{+}} \mathrm{U} 87$ and control CD133+ U87/GFP cells (Fig. 6A and B). These results suggest that the NEAT1/miR-107/CDK6 axis may also exist in U87 GSCs.

\section{Discussion}

Glioma is a highly fatal disease and a major contributor to death in patients with central nervous system tumors $(2,3,5,26)$. Glioma is asymptomatic in the early stage and is generally detected with extensive invasion into adjacent normal brain tissue (26). Accumulating evidence shows that glioma stem cells (GSCs) are responsible for tumor initiation, relapse and therapeutic resistance (27-30). Thus, novel therapeutic approaches targeting GSCs are urgently needed. Recently, several studies have demonstrated the regulation of specific lncRNAs in cancer biology including proliferation, apoptosis, tumor metabolism and invasiveness (9,31-34). Among these IncRNAs, NEAT1 has earned the reputation as a transcriptional regulator for numerous genes. Neat1 plays an important role in maintaining the integrity of subnuclear paraspeckles (35). NEAT1 regulates HIF-2 $\alpha$ in breast cancer cells under hypoxia (36). NEAT1 regulates expression of prostate cancer genes by altering the epigenetic landscape at target gene promoters to favor transcription (13). NEAT1 induces tumor cell migration and invasion in ESCC (10). Moreover, the miR-449/NEAT1 axis exists in lung cancer cells regulating the cell growth and apoptosis of lung cancer cell lines (37). In gliomas, NEAT1 has been reported to be correlated to larger tumor size, higher WHO grade and recurrence $(12,16,24)$. NEAT1 upregulates c-MET promoting 
cell growth in gliomas (24). However, its possible role in GSCs has never been explored. Previous studies in our laboratory have shown that NEAT1 knockdown can also induce the apoptosis of ESCC cells (10). These findings prompted us to evaluate the possible role of NEAT1 in GSCs using the same siRNAs of NEAT1/scramble siRNA system.

In the present study, we first evaluated the NEAT1 levels in 29 human glioma tissues (5 WHO II, 11 WHO III, 13 WHO IV grade). We found higher NEAT1 levels in WHO grade III and IV glioma tissues, which is consistent with previous studies $(12,16)$. However, there was no difference in NEAT1 levels between WHO grade III and IV glioma tissues. This observation may be due to the deviation during the process of obtaining samples from specimens, since the NEAT1 levels in glioma may be not homogeneous. Moreover, the number of samples was too small to show the real state. More samples are needed to determine this issue. In addition, many researchers obtained RNA from wax-embedded tissue specimen. RNA may be contaminated and damaged during the different steps of the processing of tissue sections. Freshly resected glioma tissue is recommended for further research.

To examine the NEAT1 role in GSCs, we first tried to isolate GSCs from human glioma samples. There are many markers used to identify and isolate stem cells in glioma, including CD133, Nestin, CD15, Sox 2 and Msi-1 (6,7). At present, CD133 remains the most generally accepted marker for GSC isolation $(19-22,38,39)$. Although no single marker has been shown to be sufficient to confer stem cell-like properties, most $\mathrm{CD}_{133^{+}}$cells in GBM exhibit stem cell properties $(20,22,38)$. In a previous study in our laboratory, we isolated cancer stem cells from prostate cancer cells and GSCs using microbead selection $(17,23)$. In the present study, we used microbeads to isolate CD133 and nestin GSCs. However, due to our limited isolation technologies, we only successfully isolated CD133 ${ }^{+}$ cells from human glioma primary culture cells. As nestin is another well accepted marker for GSCs (20), we also examined nestin expression in the $\mathrm{CD} 133^{+}$cells isolated from our human glioma primary culture cells. We found that most of the $\mathrm{CD}_{133^{+}}$cells were also nestin-positive cells. In addition, the $\mathrm{CD}_{133^{+}}$cells from human glioma primary culture cells still exhibited stem cell properties including glioma sphere formation, glioma sphere proliferation/generation and cell differentiation. After isolation, we found that $\mathrm{CD}_{133}{ }^{+} \mathrm{GSCs}$ had higher NEAT1 levels than the human glioma primary culture cells. This observation suggests the NEAT1 may play a role in maintaining glioma stem cell properties.

Based on these observation, we further investigated the NEAT1 influence on the GSCs. We first tried to use GSCs from primary culture of glioma tissue. However, we found that after NEAT1 knockdown was carried out in GSCs from the primary culture cells, most of the GSCs were unable to generate glioma sphere and many cells grew into differentiated cells. These indicates that NEAT1 is needed for maintained stemness. Another explanation for these phenomena may be our immature GSC transfection technology. However, as aforementioned, we also found that the transfection efficiency in GSCs from different patients varied greatly. Due to the great heterogeneity, we isolated GSCs from U87 glioma cells to obtain homogeneity. After isolation and confirmation, we transfected U87 GSCs with siNEAT1 or scramble siRNA in serum-free medium. We found that NEAT1 knockdown reduced cell proliferation. NEAT1 knockdown also reduced the speed of glioma sphere formation, and some of the NEAT1-knockdown cells grew into differentiated cells. This observation is in accordance with that of the $\mathrm{CD}_{133^{+}}$human glioma primary culture cells, indicating the possible role of NEAT1 in maintaining stemness in GSCs.

A previous study showed that NEAT1 can modulate CDK6 through miR-107, which in turn regulates the cell cycle in human laryngeal squamous cell cancer (15). We found decreased CDK6 protein levels and increased miR-107 RNA levels in the NEAT1-knockdown cells. These results suggest the existence of the NEAT1/miR-107/CDK6 axis in GSCs. Since CDK6 is a regulator of the cell cycle, we further examined the cell cycle in NEAT1-knockdown cells. We found G1 phase arrest in most of the NEAT1-knockdown GSCs. This may explain the reason why NEAT1 knockdown reduced the proliferation of GSCs and promoted GSC to grow into differentiated cells.

To date, most patients with glioma receive combined therapy with little efficacy (2). Since GSCs are responsible for tumor maintenance, recurrence and resistance to conventional treatments, therapy targeting NEAT1 may hold great promise for glioma treatment $(5,26)$. In the present study, we found that GSCs had higher NEAT1 levels than the relative differentiated cells. We demonstrated for the first time that NEAT1 may play a role in maintaining stemness in GSCs. Consistent with previous study, NEAT1-knockdown cells displayed increased miR-107 RNA levels and decreased CDK6 protein levels. These verified the NEAT1/miR-107/CDK6 axis in GSCs, indicating that NEAT1 may modulate CDK6 by miR-107, which in turn regulates the cell cycle in GSCs. Previous studies showed that NEAT1 altered the epigenetic landscape of target gene promoters $(11,15,36,37)$. However, in addition to the miR-107/ CDK6 axis, there must be many other target genes regulated by NEAT1, which need further study.

There are many inherent limitations in the present study. We only isolated GSCs via CD133 selection. Previous data advocate several cell markers for GSCs. Although in accordance with previous studies $(19,20,22), \mathrm{CD}_{133^{+}}$human glioma primary culture and CD133+ ${ }^{+} 87$ cancer stem cells exhibit GSC properties, further cell marker selection may improve the purity and stemness of the isolated GSCs. NEAT1 has been reported to alter many gene promoters, which regulate cancer biology (36). However, in addition to the miR-107/ CDK6 axis, many other target genes are needed to be explored by further research. In addition, the present study is lacking direct evidence in vivo, which is ongoing in our laboratory. Nevertheless, the present study demonstrated for the first time that GSCs have higher NEAT1 levels than the relative differentiated cells and NEAT1 may play a role in maintaining stemness in GSCs. The NEAT1/miR-107/CDK6 axis may be involved in mediating the cell cycle of GSCs. The signaling pathway involved in the regulation of cancer biology by NEAT1 in GSCs warrants further exploration.

\section{Acknowledgements}

The present study was supported by joint funds of The National Natural Science foundation of China (grant no. U1404822). 


\section{References}

1. Khan IS and Ehtesham M: Emerging strategies for the treatment of tumor stem cells in central nervous system malignancies. Adv Exp Med Biol 853: 167-187, 2015.

2. Chien LN, Ostrom QT, Gittleman H, Lin JW, Sloan AE, Barnett GH, Elder JB, McPherson C, Warnick R, Chiang YH, et al: International differences in treatment and clinical outcomes for high grade glioma. PLoS One 10: e0129602, 2015.

3. Mannas JP, Lightner DD, Defrates SR, Pittman T and Villano JL: Long-term treatment with temozolomide in malignant glioma. J Clin Neurosci 21: 121-123, 2014.

4. Perry A and Wesseling P: Histologic classification of gliomas. Handb Clin Neurol 134: 71-95, 2016.

5. Reulen HJ, Poepperl G, Goetz C, Gildehaus FJ, Schmidt M, Tatsch K, Pietsch T, Kraus T and Rachinger W: Long-term outcome of patients with WHO Grade III and IV gliomas treated by fractionated intracavitary radioimmunotherapy. J Neurosurg 123: 760-770, 2015.

6. Modrek AS, Bayin NS and Placantonakis DG: Brain stem cells as the cell of origin in glioma. World J Stem Cells 6: 43-52, 2014

7. Dietrich J, Diamond EL and Kesari S: Glioma stem cell signaling: Therapeutic opportunities and challenges. Expert Rev Anticancer Ther 10: 709-722, 2010.

8. Qi P and Du X: The long non-coding RNAs, a new cancer diagnostic and therapeutic gold mine. Mod Pathol 26: 155-165, 2013.

9. Li X, Wu Z, Fu X and Han W: Long noncoding RNAs: Insights from biological features and functions to diseases. Med Res Rev 33: 517-553, 2013.

10. Chen X, Kong J, Ma Z, Gao S and Feng X: Up regulation of the long non-coding RNA NEAT1 promotes esophageal squamous cell carcinoma cell progression and correlates with poor prognosis. Am J Cancer Res 5: 2808-2815, 2015.

11. Li Y, Li Y, Chen W, He F, Tan Z, Zheng J, Wang W, Zhao Q and Li J: NEAT expression is associated with tumor recurrence and unfavorable prognosis in colorectal cancer. Oncotarget 6 : 27641-27650, 2015.

12. He C, Jiang B, Ma J and Li Q: Aberrant NEAT1 expression is associated with clinical outcome in high grade glioma patients. APMIS 124: 169-174, 2016.

13. Chakravarty D, Sboner A, Nair SS, Giannopoulou E, Li R, Hennig S, Mosquera JM, Pauwels J, Park K, Kossai M, et al: The oestrogen receptor alpha-regulated lncRNA NEAT1 is a critical modulator of prostate cancer. Nat Commun 5: 5383, 2014.

14. Blume CJ, Hotz-Wagenblatt A, Hüllein J, Sellner L, Jethwa A, Stolz T, Slabicki M, Lee K, Sharathchandra A, Benner A, et al: p53-dependent non-coding RNA networks in chronic lymphocytic leukemia. Leukemia 29: 2015-2023, 2015.

15. Wang $\mathrm{P}, \mathrm{Wu} \mathrm{T}$, Zhou $\mathrm{H}$, Jin Q, He G, Yu H, Xuan L, Wang X, Tian L, Sun Y, et al: Long noncoding RNA NEAT1 promotes laryngeal squamous cell cancer through regulating miR-107/CDK6 pathway. J Exp Clin Cancer Res 35: 22, 2016.

16. Liu Q, Sun S, Yu W, Jiang J, Zhuo F, Qiu G, Xu S and Jiang X: Altered expression of long non-coding RNAs during genotoxic stress-induced cell death in human glioma cells. J Neurooncol 122: 283-292, 2015.

17. Wang X, Ma Z, Xiao Z, Liu H, Dou Z, Fengxand Shi H: Chk1 knockdown confers radiosensitization in prostate cancer stem cells. Oncol Rep 28: 2247-2254, 2012.

18. Chen W, Xiao Z, Zhao Y, Huang L and Du G: HIF-1 $\alpha$ inhibition sensitizes pituitary adenoma cells to temozolomide by regulating MGMT expression. Oncol Rep 30: 2495-2501, 2013.

19. Lathia JD, Hitomi M, Gallagher J, Gadani SP, Adkins J, Vasanji A, Liu L, Eyler CE, Heddleston JM, Wu Q, et al: Distribution of CD133 reveals glioma stem cells self-renew through symmetric and asymmetric cell divisions. Cell Death Dis 2: e200, 2011.

20. Dahlrot RH, Hansen S, Jensen SS, Schrøder HD, Hjelmborg J and Kristensen BW: Clinical value of CD133 and nestin in patients with glioma: A population-based study. Int J Clin Exp Pathol 7: 3739-3751, 2014.
21. Clément V, Dutoit V, Marino D, Dietrich PY and Radovanovic I: Limits of CD133 as a marker of glioma self-renewing cells. Int J Cancer 125: 244-248, 2009.

22. Griguer CE, Oliva CR, Gobin E, Marcorelles P, Benos DJ, Lancaster JR Jr and Gillespie GY: CD133 is a marker of bioenergetic stress in human glioma. PLoS One 3: e3655, 2008.

23. Wu J, Lai G, Wan F, Xiao Z, Zeng L, Wang X, Ye F and Lei T: Knockdown of checkpoint kinase 1 is associated with the increased radiosensitivity of glioblastoma stem-like cells. Tohoku J Exp Med 226: 267-274, 2012.

24. Zhen L, Yun-Hui L, Hong-Yu D, Jun M and Yi-Long Y: Long noncoding RNA NEAT1 promotes glioma pathogenesis by regulating miR-449b-5p/c-Met axis. Tumour Biol 37: 673-683, 2016.

25. Rader J, Russell MR, Hart LS, Nakazawa MS, Belcastro LT, Martinez D, Li Y, Carpenter EL, Attiyeh EF, Diskin SJ, et al: Dual CDK4/CDK6 inhibition induces cell-cycle arrest and senescence in neuroblastoma. Clin Cancer Res 19: 6173-6182, 2013.

26. Brzozowska A, Toruń A and Mazurkiewicz M: The impact of surgery on the efficacy of adjuvant therapy in glioblastoma multiforme. Adv Clin Exp Med 24: 279-287, 2015.

27. Jung J, Gilbert MR and Park DM: Isolation and propagation of glioma stem cells from acutely resected tumors. Methods Mol Biol 1516: 361-369, 2016.

28. Codrici E, Enciu AM, Popescu ID, Mihai S and Tanase C: Glioma stem cells and their microenvironments: Providers of challenging therapeutic targets. Stem Cells Int 2016: 5728438, 2016.

29. Liebelt BD, Shingu T, Zhou X, Ren J, Shin SA and Hu J: Glioma stem cells: Signaling, microenvironment, and therapy. Stem Cells Int 2016: 7849890, 2016.

30. Kim SS, Pirollo KF and Chang EH: Isolation and culturing of glioma cancer stem cells. Curr Protoc Cell Biol 67: 23.10.1-23.10.10, 2015. doi: 10.1002/0471143030.cb2310s67.

31. Han L, Zhang K, Shi Z, Zhang J, Zhu J, Zhu S, Zhang A, Jia Z, Wang G, Yu S, et al: LncRNA profile of glioblastoma reveals the potential role of lncRNAs in contributing to glioblastoma pathogenesis. Int J Oncol 40: 2004-2012, 2012.

32. Sørensen KP, Thomassen M, Tan Q, Bak M, Cold S, Burton M, Larsen MJ and Kruse TA: Long non-coding RNA expression profiles predict metastasis in lymph node-negative breast cancer independently of traditional prognostic markers. Breast Cancer Res 17: 55, 2015.

33. Zhang X, Sun S, Pu JK, Tsang AC, Lee D, Man VO, Lui WM, Wong ST and Leung GK: Long non-coding RNA expression profiles predict clinical phenotypes in glioma. Neurobiol Dis 48: $1-8,2012$.

34. Li R, Qian J, Wang YY, Zhang JX and You YP: Long noncoding RNA profiles reveal three molecular subtypes in glioma. CNS Neurosci Ther 20: 339-343, 2014.

35. Clemson CM, Hutchinson JN, Sara SA, Ensminger AW, Fox AH, Chess A and Lawrence JB: An architectural role for a nuclear noncoding RNA: NEAT1 RNA is essential for the structure of paraspeckles. Mol Cell 33: 717-726, 2009.

36. Choudhry H, Albukhari A, Morotti M, Haider S, Moralli D, Smythies J, Schödel J, Green CM, Camps C, Buffa F, et al: Tumor hypoxia induces nuclear paraspeckle formation through HIF-2 $\alpha$ dependent transcriptional activation of NEAT1 leading to cancer cell survival. Oncogene 34: 4546, 2015.

37. You J, Zhang Y, Liu B, Li Y, Fang N, Zu L, Li X and Zhou Q: MicroRNA-449a inhibits cell growth in lung cancer and regulates long noncoding RNA nuclear enriched abundant transcript 1. Indian J Cancer 51 (Suppl 3): e77-e81, 2014.

38. Campos B and Herold-Mende CC: Insight into the complex regulation of CD133 in glioma. Int J Cancer 128: 501-510, 2011.

39. Zhang M, Song T, Yang L, Chen R, Wu L, Yang Z and Fang J: Nestin and CD133: Valuable stem cell-specific markers for determining clinical outcome of glioma patients. J Exp Clin Cancer Res 27: 85, 2008. 